\title{
Cosmic-Ray Positive and Negative Electrons
}

\author{
Carl D. Anderson, California Institute of Technology
}

(Received June 19, 1933)

\begin{abstract}
A determination of the specific ionization of cosmic-ray particles, first, by a count of the number of drops per $\mathrm{cm}$ along cosmic-ray tracks on cloud-chamber photographs and, second, by measurements of the energy loss in lead has shown that the great bulk of the cosmic-ray particles of positive charge are positive electrons. The primary ionization was found to be about 31 ion-pairs per $\mathrm{cm}$ in air at S.T.P., but the total energy loss represents about 120 ion pairs per $\mathrm{cm}$ in air. Approximately the same values of specific ionization were found for the positives as for the negatives. Positive and negative electrons were found to occur in nearly equal numbers and to have similar distributions in energy. Energy distribution curves are given for the positives and negatives. A brief description of the experimental procedure is given, and several track photographs are shown. In view of the discovery that
\end{abstract}

hard gamma-rays of Th $C^{\prime \prime}$ give rise to positives and negatives in pairs, similar to the effects found in cosmicray studies, it is concluded that the absorption of the Th $C^{\prime \prime}$ rays is due in part to that by free negative electrons and in part to a nuclear effect which results in the production of pairs of positive and negative electrons, the former effect accounting for the greater part of the absorption of the $T h C^{\prime \prime}$ rays. The symmetry in occurrence of the positive and negative electrons found in the cosmicray studies shows that the nuclear effect which results in the production of positive and negative electrons in pairs represents the predominant part of the absorption for the range of energies as high as those of the cosmic rays and that the absorption by free negative electrons is relatively small.
$T$ HE Wilson cloud-chamber photographs of Skobelzyn ${ }^{1}$ showed the existence of cosmicray particles of energy greater than $15 \times 10^{6}$ electron-volts, his limit of measurement.

Experiments ${ }^{2}$ by the writer in collaboration with Professor R. A. Millikan making use of a vertical Wilson chamber, specially designed for a study of cosmic rays and capable of measuring energies up to 3 and $4 \times 10^{9}$ volts by means of a strong magnetic field up to 20,000 gauss brought to light the fact that there occur particles of negative charge and also particles of positive charge in abundance. A preliminary survey of their energies was given.

By measurements on the density of ionization along the track it was shown that in general the tracks of both positive and negative curvatures were due to particles of charge equal to that of one electron, and could not be due to particles of multiple charge.

For the purpose of studying in detail the mechanics of the interaction of the particles with

\footnotetext{
${ }^{1}$ Skobelzyn, Zeits. f. Physik 54, 686 (1929).

${ }_{2}$ Millikan and Anderson, Phys. Rev. 40, 325 (1932), and Anderson, Phys. Rev. 41, 405 (1932). See Kunze, Zeits. f. Physik 80, 559 (1933).
}

matter and to help identify the particles of positive charge, a sheet of lead was inserted in the chamber so as to allow the observation of the particles before and after they penetrated the lead plate. It was then possible to study the scattering and energy loss of the particles in lead, ${ }^{3}$ and in particular, effects were obtained which we could interpret logically on the basis of specific ionization, curvature, and range as due only to particles of positive charge and mass small compared with that of the proton. ${ }^{4}$ These particles have been called positrons. Blackett and Occhialini, using a tube-counter controlled Wilson chamber in a magnetic field of 3000 gauss, obtained effects similar to those that we observed and confirmed our conclusion that it is necessary to call upon positive particles of mass small compared with that of the proton to explain the observed effects. ${ }^{5}$

The tendency of the cosmic-ray particles to occur in groups, first noticed by Skobelzyn, also

\footnotetext{
${ }^{3}$ Anderson, Phys. Rev. 43, $381 \mathrm{~A}$ (1933).

${ }^{4}$ Anderson, Science 76, 238 (1932) and Phys. Rev. 43, 491 (1933).

${ }^{5}$ Blackett and Occhialini, Proc. Roy. Soc. A139, 699 (1933).
} 
a marked feature of our photographs, ${ }^{6}$ is strongly emphasized in the photographs of Blackett and Occhialini.

Before the lead plate was inserted in the chamber we interpreted the tracks of negative curvature as due to electrons, and the tracks of positive curvature as due probably to protons, at that time the only known positive particle of unit charge. This interpretation was made with some hesitation, however, because of the fact that while protons and electrons at high energies produce about the same ionization the positive tracks of considerable curvature could only with difficulty be ascribed to protons because of the meager ionization exhibited. Fig. 2 shows in a general way the ionization of protons as compared to particles of the same charge but of a mass equal to that of the free electron as a function of the curvature in the magnetic field. Fig. 3 gives for comparison the energy of protons and electrons for various values of $H \rho$. We pointed out at that time that precise data on the specific ionization of the lower energy positive tracks were needed to distinguish protons from electrons. One of the purposes of this paper is to report the results of this work, which lead to the conclusion that the bulk of the positive particles are positrons and not protons. Additional evidence for this conclusion will be brought out in a discussion of the curvature distribution of the positive and negative tracks.

\section{Experimental Procedure}

In answer to numerous queries a brief description will be given of the experimental method employed. A vertical Wilson chamber of $16.5 \mathrm{~cm}$ diameter and $4 \mathrm{~cm}$ depth was incorporated in a large electromagnet designed to produce a uniform magnetic field over the volume of the chamber of magnitude sufficient to measure the energies to be expected for the cosmic-ray particles. The magnet contains 800 turns of copper tubing of $\frac{1}{4}$ inch inside and $\frac{3}{8}$ inch outside diameter wound in the form of 40 pies and arranged in two coils. The copper tubing serves the double purpose of carrying the electric current and the circulating water for cooling purposes, the pies in each coil being connected in series

${ }^{6}$ Anderson, Phys. Rev. 43, 368 (1933). electrically and in parallel for the water circuits. For the greater part of the work a current of 1600 amperes at a potential of 275 volts was used, the two coils being connected in parallel and giving a field of 15,000 gauss. A steady flow of about 35 gallons of water per minute through the coils was maintained during operation. A schematic sketch indicating the relationship of the essential parts is shown in Fig. 1. Located at the center of

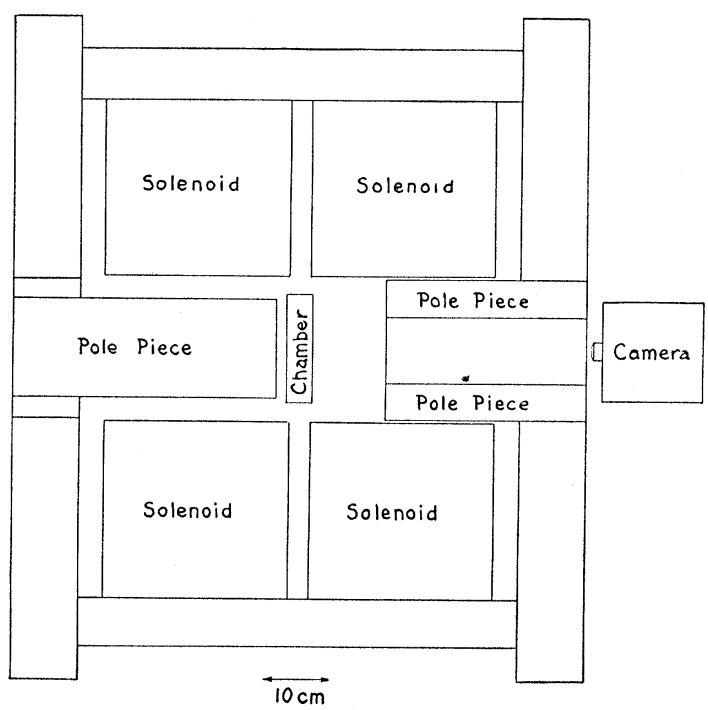

FIG. 1. Schematic diagram of apparatus.

the coils is the Wilson chamber. It is of the reciprocating piston type, a thin rubber diaphragm placed across the head of the piston sealing it air-tight. It operates automatically at the rate of one expansion every 14 seconds. The air is drawn out from behind the piston to produce the expansion. Nearly filling the space inside the piston is a solid core of Armco iron of $14.8 \mathrm{~cm}$ diameter. Separated from this by an air-gap of $16.5 \mathrm{~cm}$ is the second pole piece of 22 $\mathrm{cm}$ diameter which contains a square hole $11 \mathrm{~cm} \times 11 \mathrm{~cm}$ through which the tracks are photographed. The magnetic field is uniform to within 10 percent throughout the volume occupied by the chamber. The cloud chamber suffers practically no temperature increase during a run which is usually of two hours' duration, and 500 photographs are taken without an adjustment of the expansion ratio. A carbon arc light is used for illumination, and the photographs are taken 
on $35 \mathrm{~mm}$ supersensitive panchromatic film. A beam of light collimated to a width of $1 \mathrm{~cm}$ was used for most of the work. A single photograph was considered sufficient to give the data desired, though by a simple arrangement provision has been made for taking stereoscopic pictures. A pair of mirrors was placed at the sides of the square hole in the iron pole piece through which the photographs were taken, and the two reflected images of the chamber along with the central direct central image were all recorded together on the one film. See for example Fig. 18.

The yield of cosmic-ray tracks is in agreement with that to be expected from the amount of ionization due to cosmic radiation as measured in ionization chambers. Including short tracks and the very diffuse tracks, about one track per five expansions is obtained. To date over 1500 photographs of cosmic-ray tracks have been obtained. Of course, only the long and sharp tracks which represent but a fraction of these are suitable for curvature measurements. For the purpose of determining the distribution in energy of the particles, given in this paper, only the long and exceedingly sharp tracks were chosen. Extreme care was taken to eliminate to a high degree all disturbing influences such as motion of the gas in the chamber, falling drops, etc. To insure against errors due to falling drops the camera shutter was opened just previous to the time of formation of the drops; thus any motion of the drops would appear on the photographs and could not escape detection. The tracks from which the energy distribution was determined were particularly free from such effects.

\section{SPECIFIC IONIZATION}

In order to determine the abundance of positrons as compared with protons the specific ionization was determined for a number of tracks of both positive and negative curvature by counting on diffuse tracks the number of drops per $\mathrm{cm}$. (See for example Figs. 6 and 7.) By this method differences in specific ionization of 20 percent could readily be detected. The difference in specific ionization between a positron and proton amounts to about 20 percent for a value of $H \rho$ equal to $3 \times 10^{6}$ gauss-cm, and for curvatures greater than this the difference becomes more pronounced until at values of $H \rho$ less than $10^{6}$ gauss-cm the ionization of positrons and protons differs in order of magnitude (see Fig. 2). Al-

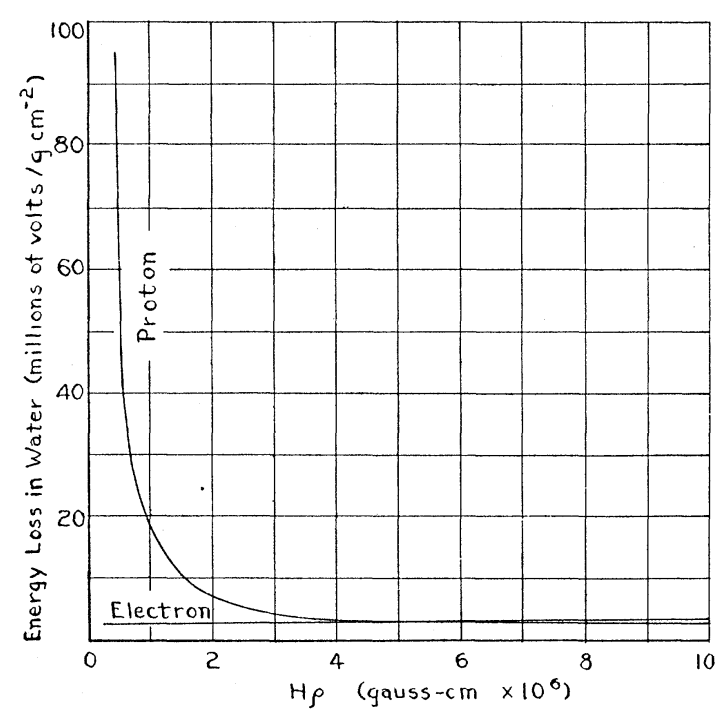

FIG. 2. Relative ionization of protons and electrons in terms of curvature in magnetic field.

though for tracks of the degree of diffuseness used for drop-counts it is possible to measure energies only for the more curved tracks, it is these which are best suited for differentiating between positrons and protons. Those of positive curvature do not in general show a greater ionization than those of negative curvature, and the amount of ionization measured is consistent with that to be expected from particles of small mass (of the order of that of the free electron) and for the more curved tracks is definitely inconsistent with that to be expected from protons. Further, for a large number of slightly diffuse positively curved tracks where the ions are not sufficiently resolved for an exact count, but where a difference in ionization such as that to be expected between positrons and protrons could readily have been detected, positrons rather than protons were indicated for the bulk of the tracks. For individual cases of single positively curved tracks it is not possible to state definitely that the tracks represent a positive particle travelling downward through the chamber rather than a negative particle travelling upward. However, it is a safe inference based on various kinds of evi- 
dence which we have previously pointed out that the tracks of positive curvature in general represent downward positives rather than upward negatives. Professor Millikan and Dr. Neher have found that a screen of lead placed above their electroscope decreases the ionization by a considerable amount whereas the same lead screen below the electroscope produces no detectable change in ionization. This also is strong evidence that the particles are in general moving downward. The conclusion is reached, therefore, that the positives in general are positrons rather than protons, though in two cases we have found tracks which ionize too heavily to represent electrons and may represent protons. Blackett and Occhialini ${ }^{5}$ have also reported protons but conclude that the positives in general represent positrons. Heavier positive particles may also occur but because of their relatively short range may frequently escape detection.

The drop-counts on 26 diffuse tracks lead to a mean value of 31 ion-pairs per $\mathrm{cm}$ in air at S.T.P. which is in fair accord with the value of 36 ionpairs reported by Locher. ${ }^{7}$ This must, however, represent only a lower limit to the actual specific ionization because of clusters of large numbers of ions at various points along the tracks at which the ions could not be accurately counted and were therefore not included in the count. Occasionally an electron will be removed from the $k$-shell of an atom of the gas through which the particle passes, and in returning to its normal state the atom by internal conversion of the photon may emit an electron which carries off the ionization energy. An electron of this energy would have only a very short range and the ionization would all be produced in the immediate neighborhood of the track. An electron may also receive a considerable energy by a close encounter and because of the presence of the strong magnetic field be sufficiently coiled up so as to produce ionization only in the immediate neighborhood of the track (unless the energy received is of the order of a million volts or more, an event which is relatively improbable). A study of these effects on the mean energy loss of the particles is now being made. Kolhorster and Tuwin ${ }^{8}$ by combined electroscope and Geiger-counter deter-

${ }^{7}$ Locher, Phys. Rev. 39, 883 (1932).

${ }^{8}$ Kolhorster and Tuwin, Zeits. f. Physik 81, 435 (1933). minations report a value of 135 ion-pairs per $\mathrm{cm}$. This figure includes effects such as the two mentioned above, and this together with the effect of associated tracks may account for the high value they find.

Another method for determining the energy loss of the cosmic-ray particles is to measure their energies before and after they penetrate a plate of a given material of known thickness. We have reported a preliminary value of about $35 \times 10^{6}$ volts per cm energy loss in lead for electrons of the order of $300 \times 10^{6}$ volts energy. ${ }^{3}$ The energy loss of particles in such a lead plate is subject to considerable statistical fluctuation and further measurements will be needed before an accurate mean value of energy can be given. Fig. 8 shows an electron of 113 million volts initial energy which loses 27 million volts in passing through $13.4 \mathrm{~mm}$ of lead. This corresponds to an energy loss close to 20 million volts per $\mathrm{cm}$ which is good accord with the calculated value of 19.4 million volts per $\mathrm{cm}$ of lead for electrons of 100 million volts energy given by Bethe. ${ }^{9}$ The mean value for energy loss in lead on the basis of our present data is, however, greater than this and is approximately 35 million volts per $\mathrm{cm}$.

For comparison with specific ionization measured in air it is interesting to compute the number of ion-pairs per $\mathrm{cm}$ to be expected in air on the basis of a value of 20 million volts per $\mathrm{cm}$ in lead. Using the values given by Bethe for the relative energy loss per $\mathrm{g} \mathrm{cm}^{-2}$ in water and lead (2.90 million volts per $\mathrm{g} \mathrm{cm}^{-2}$ in water as compared to 1.76 million volts per $\mathrm{g} \mathrm{cm}^{-2}$ in lead for 100 million volt-electrons) and assuming the same value per $\mathrm{g} \mathrm{cm}^{-2}$ for air as for water, we find an energy loss of 3900 volts per $\mathrm{cm}$ for air at S.T.P. If we take a mean value of 32 volts as the energy required to produce a pair of ions, ${ }^{10}$ this leads to 122 ion-pairs per $\mathrm{cm}$ in air at S.T.P. It appears probable that though the primary ionization is 30 to 35 ion-pairs per $\mathrm{cm}$ in air, the total ionization is of the order of $120-140$ ion-pairs per $\mathrm{cm}$ for electrons in this energy range.

Although drop-counts have shown no differ-

${ }^{9}$ Bethe, Zeits. f. Physik 76, 293 (1932). See also Carlson and Oppenheimer, Phys. Rev. 41, 763 (1932).

${ }^{10}$ Kulenkampff, Phys. Zeits. 30, 777 (1929). 
ence in ionization between positives and negatives, the energy loss in lead for the positives is slightly higher than for the negatives as far as the data have been collected. This may well be a statistical fluctuation and further work is in progress.

\section{Curvature Measurements}

We have given the results of energy measurements for a number of tracks, ${ }^{11}$ and Kunze ${ }^{12}$ has reported energies in essential agreement with ours. Curvature measurements have now been made on a new set of very carefully selected tracks. A curvature distribution based on a relatively small number of tracks on most of which accurate measurement was possible was considered preferable to one based on a larger number of tracks which included short and diffuse tracks. Hence only very sharp tracks longer than $8 \mathrm{~cm}$ were chosen. Precautions were taken to reduce to a minimum all disturbing influences in the cloud chamber which might introduce errors. Figs. 13-17 are typical examples of tracks of this group. Measurements were made on the original negatives by means of a comparator, the coordinates of points $0.5 \mathrm{~mm}$ apart being measured and plotted. In Fig. 4 is plotted a typical set of such measurements. The units of ordinates are millimeters and the units of abscissae are hundredths of millimeters as measured on the original negative film which is 0.14 full size. A circular track plotted in this way forms a parabola and from a measurement of $a$ and $b$ on the graph the radius of curvature can be determined. Electron energies up to one or two billion volts were in most cases found readily measurable.

The values of the energies of these tracks, listed separately for the positives and negatives, are given in Table I on the assumption that the positives are positrons and the negatives are electrons, an assumption justified by the evidence discussed in the previous section. These criteria for the selection of the tracks, viz., length and sharpness, should favor neither the low nor the high energies and should, therefore, give a fair cross section of the energies of the particles except for statistical fluctuations.

\footnotetext{
${ }^{11}$ Anderson, Phys. Rev. 41, 405 (1932).

${ }^{2}$ Kunze, Zeits. f. Physik 80, 559 (1933).
}

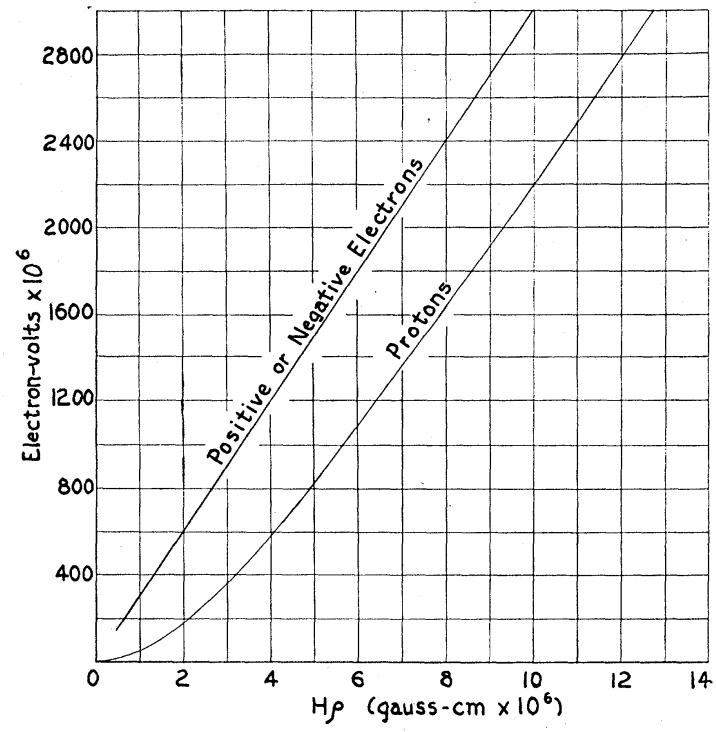

FIG. 3. Energies of fast electrons in terms of curvature in magnetic field. A curve for protons is given for comparison.

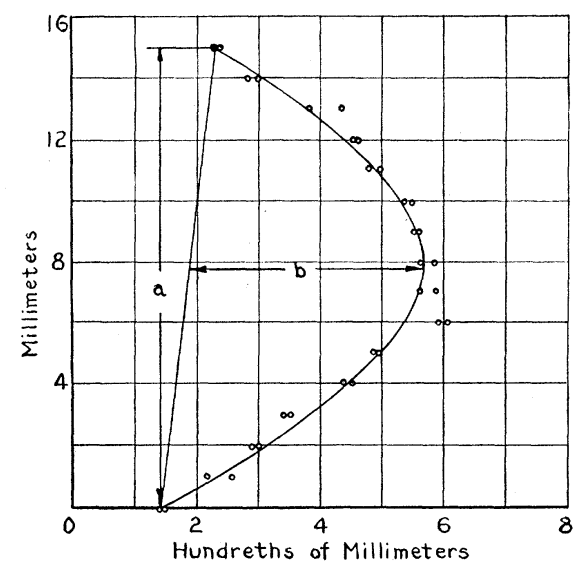

FIG. 4. Plot of the measurements of a representative high energy electron track. Radius of curvature $\left(R \sim a^{2} / 8 b\right)$ reduced to full size in chamber is $498 \mathrm{~cm}$. $H_{\rho}=7.5 \times 10^{6}$ gauss-cm; energy $=2.2 \times 10^{7}$ electron-volts.

It is to be noted first of all that in this group the positives and negatives occur in nearly equal numbers, and as Fig. 5 shows, with quite symmetrical distributions in energy.

It is necessary to point out that this energy distribution does not characterize the main cosmic-ray beam as it is incident on the earth, but only after it has been filtered by passage through the atmosphere and the screening material around the cloud chamber. The presence 
TABLE I. Energy distribution of positive and negative electrons. In addition to the tracks listed below there are to be included eleven tracks which showed irregularities due to mass-motion of the air in the cloud chamber and could not be accurately measured or classified as to sign of charge. The energies of the tracks in this group are all very probably greater than about one billion volts.

\begin{tabular}{cc|cc}
\hline $\begin{array}{c}\text { Positive } \\
\text { electrons } \\
\text { Energy } \\
\left.\text { (volts } \times 10^{6}\right)\end{array}$ & $\begin{array}{c}\text { Negative } \\
\text { electrons } \\
\text { Energy } \\
\left.\text { (volts } \times 10^{6}\right)\end{array}$ & $\begin{array}{c}\text { Positive } \\
\text { electrons } \\
\text { Energy } \\
\left.\text { (volts } \times 10^{6}\right)\end{array}$ & $\begin{array}{c}\text { Negative } \\
\text { electrons } \\
\text { Energy } \\
\left.\text { (volts } \times 10^{6}\right)\end{array}$ \\
\hline 120 & 60 & 880 & 600 \\
145 & 75 & 900 & 660 \\
166 & 85 & 970 & 800 \\
190 & 120 & 1000 & 860 \\
200 & 125 & 1000 & 870 \\
220 & 135 & 1000 & 930 \\
235 & 140 & 1150 & 1150 \\
260 & 155 & 1350 & 1250 \\
270 & 230 & 1600 & 1300 \\
310 & 280 & 1700 & 1800 \\
340 & 300 & 1900 & 2200 \\
340 & 330 & 2000 & 2400 \\
440 & 360 & 2100 & 2400 \\
480 & 370 & 2200 & 2600 \\
530 & 480 & 2400 & 2700 \\
540 & 510 & 2500 & 3000 \\
590 & 540 & 2700 & 5000 \\
640 & 540 & 2700 & \\
750 & 570 & 2700 & \\
770 & 600 & & \\
\hline \hline
\end{tabular}

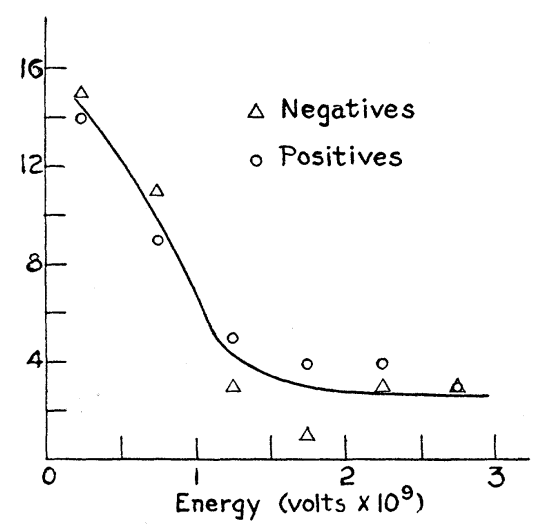

FIG. 5. Distribution in energy of the positive and negative electrons. Each point on the curve represents the number of tracks in the corresponding half-billion-volt interval.

of the large amount of heavy material, coils, pole pieces, etc., immediately surrounding the cloud chamber will also affect the energy distribution of the observed particles because of the "ubergangseffekte." Other indications point to the view that at high altitudes the cosmic-ray particles of low energies form a larger proportion than at the earth's surface.
Out of an additional group of 216 tracks which were not considered sharp enough for energy measurements but which were definitely subject to division into positive and negative curvatures, there were found 107 positives and 109 negatives indicating again approximately equal numbers of positives and negatives.

The symmetry between the positives and negatives is not maintained, however, if we consider only the low energy particles, i.e., those of curvatures corresponding to energies less than 100 million volts. For single particles of low energy because of the high degree of curvature in the magnetic field it is in general not possible to infer their sign of charge since the particles may be turned completely around by the field. Therefore a study was made only of those tracks which were associated with other tracks, where it was possible to infer their direction of motion and hence their sign of charge. In order to obtain a sufficient number with which to work it was necessary to include a number of diffuse tracks where it was not possible to determine unambiguously the direction of the curvature of the higher energy tracks, but it was always possible to determine the sign of charge and the energy of the highly curved lower energy tracks. It was here found that in 52 groups of tracks there were 64 negatives and only 5 positives in the energy range lying below 100 million volts. The excess of low energy negatives over positives associated with other tracks may well be due to close encounters between high speed particles (positive or negative) and extranuclear negative electrons in which a considerable energy transfer takes place.

The general symmetry in occurrence between the positives and negatives is in accord with the view that in the primary absorption process of the incident cosmic-ray beam positives and negatives are formed in pairs perhaps as governed by Dirac's theory of electrons. ${ }^{13}$

The near equality in the number of positive and negative particles shown here is of course only statistical. It will be noted, however, that there are 16 measured positives above one billion volts as compared to only 11 negatives. Though

${ }^{13}$ Dirac, Proc. Roy. Soc. A126, 360 (1930) and A133, 60 (1931). 


\section{Description of the Photographs}

The magnetic field strength was 15,000 gauss for all photographs except Fig. 18 where it was 650 gauss. The actual diameter of the portion of the chamber shown in the photographs is $14 \mathrm{~cm}$.

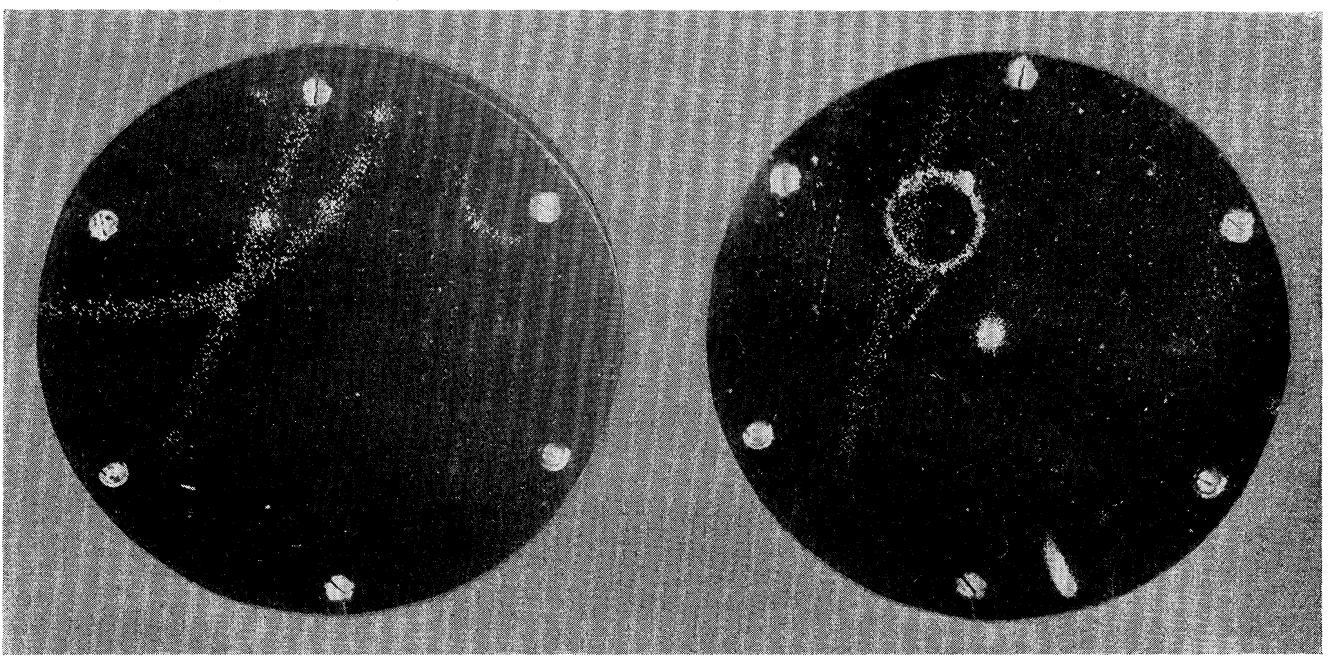

6

FIG. 6. A representative group of three "early" tracks suitable for an ion-count, showing drops resolved as a result of the diffusion of the ions. On the upper portion of the high energy track is a small cluster of ions.

FIG. 7. Three ages of tracks are represented: (1) a high energy very diffuse early track, (2) a later pair of tracks showing less diffusion of ions; the circular track is an electron of 4.8 million volts energy, the other one a higher energy track of uncertain sign of charge, and (3) at the left a short, sharp track produced by a particle which entered the chamber coincident with or immediately following the expansion.

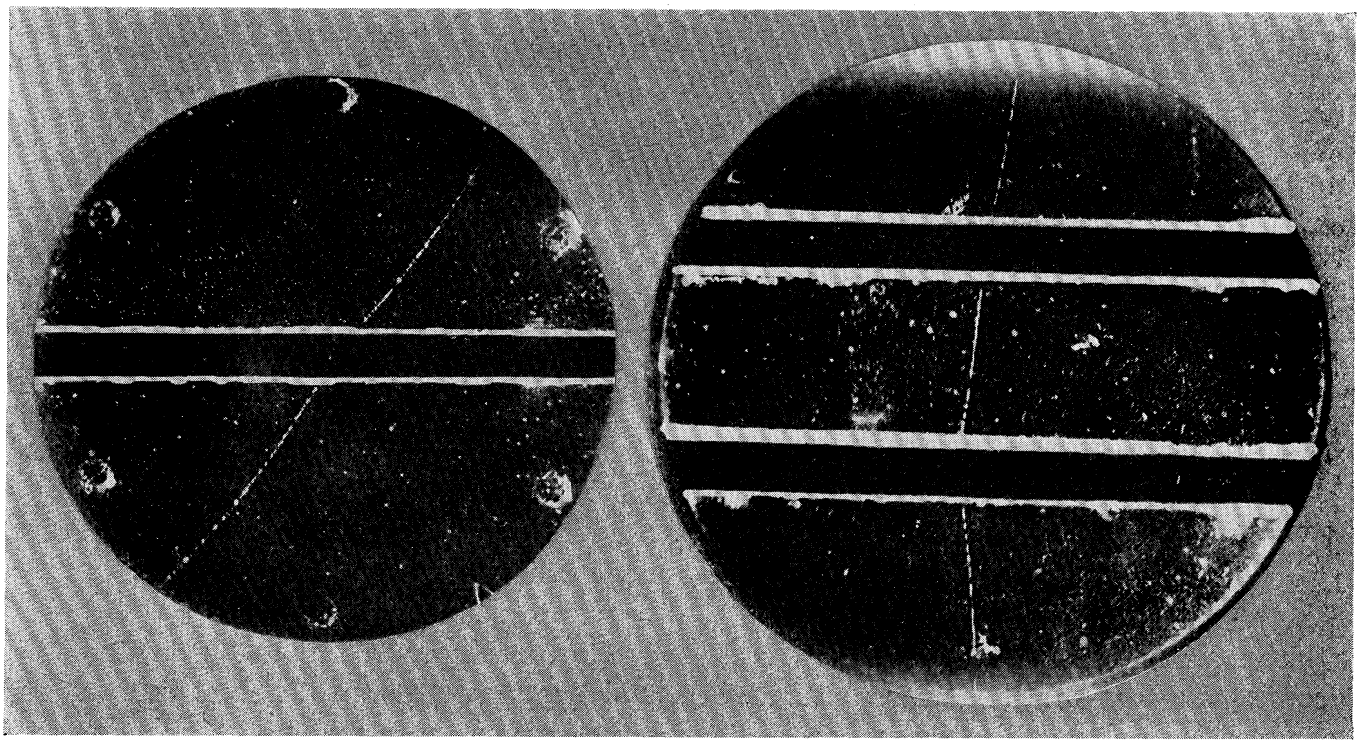

8

9

FIG. 8. A 113 million volt-electron traversed $13.4 \mathrm{~mm}$ of lead and emerged with an energy of 86 million volts, showing an energy loss of 20 million volts per $\mathrm{cm}$ of lead. Thickness of lead plate is $11 \mathrm{~mm}$.

FIG. 9. An electron of 240 million volts energy lost about 20 million volts in first passage through the $11 \mathrm{~mm}$ lead plate and an additional 60 million volts in the lower plate. 


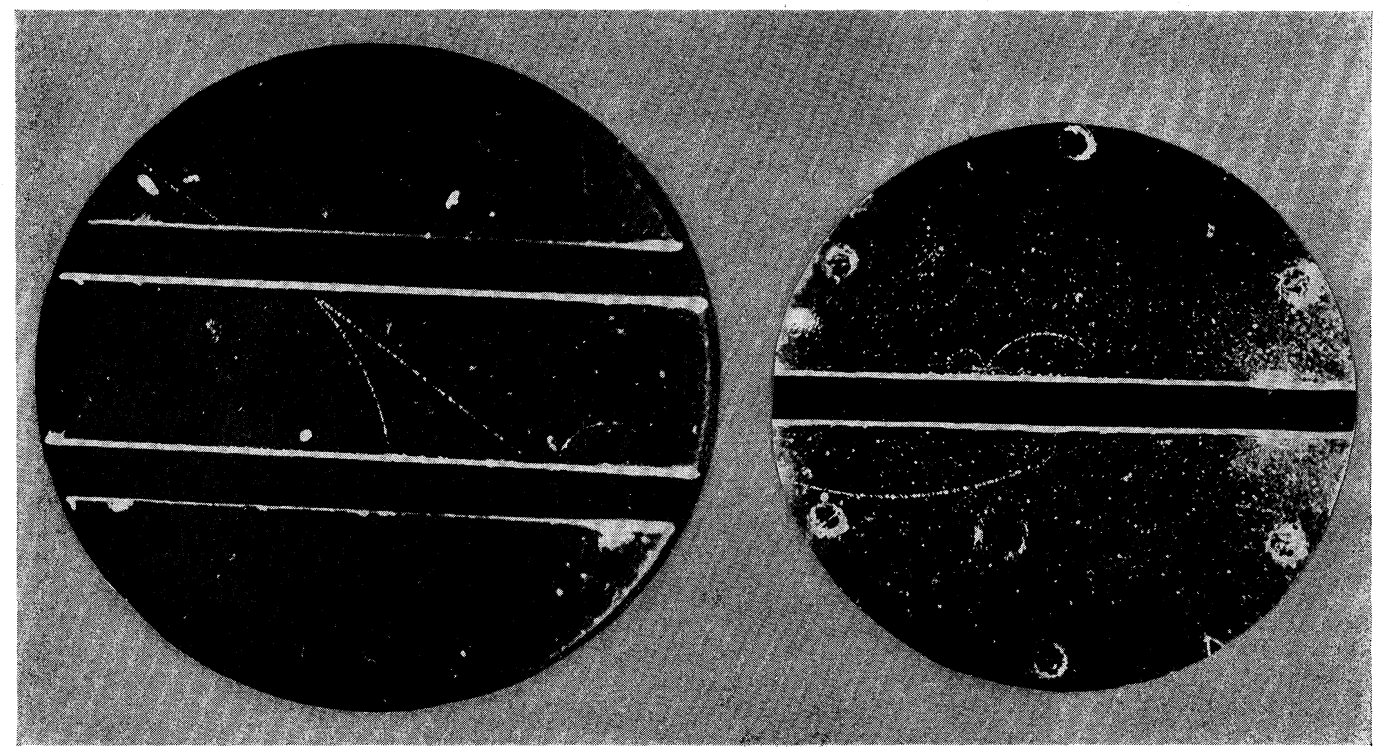

10

11

FIG. 10. A secondary electron of 32 million volts energy was ejected by a fast electron which passed through the lead plate. The angle, $\theta$, between the directions of the primary and secondary electrons satisfies within experimental uncertainty the relation $\tan \theta=\left(2 m c^{2} / E\right)^{\frac{1}{2}}$, where $E$ is the energy of the secondary electron. The energy of the primary electron measured between the plates is about 800 million volts. An additional secondary electron of about 6 million volts energy was produced in the lower plate and appears just above the lower plate at the right. The frequency of occurrence of secondary electrons produced by close encounters is in close agreement with that to be expected from theoretical considerations.

FIG. 11. There are two interpretations of this photograph. The first is that a positron of 38 million volts energy penetrated the lead plate and emerged above with 6 million volts energy. It is seen to suffer a sharp deflection upon striking the upper surface of the lead plate. The second interpretation is that an electron of 38 million volts energy was ejected downward from the plate, and at the same time a positron of 6 million volts energy ejected upward and deflected by the magnetic field until it struck the plate and rebounded. Either of these two views requires the positron interpretation.

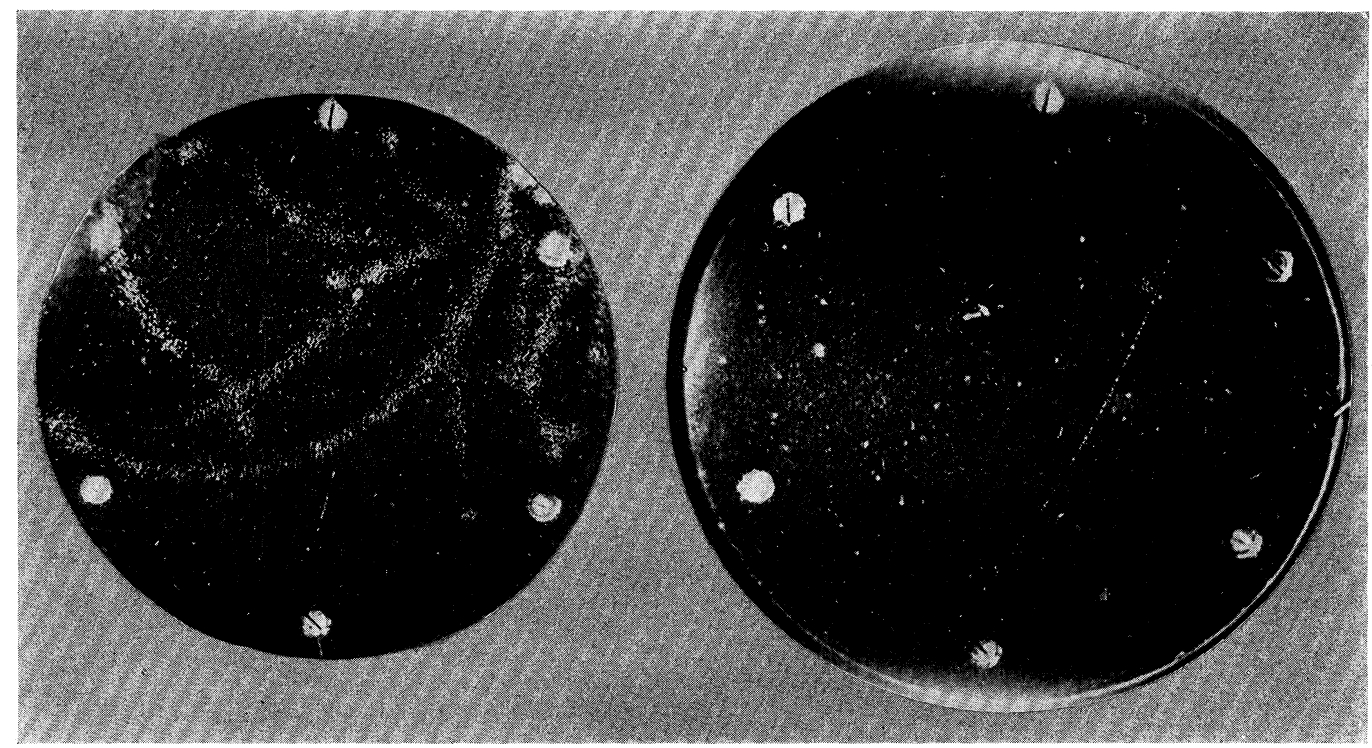

FIG. 12. A shower represented by a group of simultaneous early tracks. From the specific ionization and curvature in the magnetic field it is necessary to ascribe most of these tracks to particles of small mass, i.e., positrons or electrons.

FIG. 13. An electron of 120 million volts energy. 


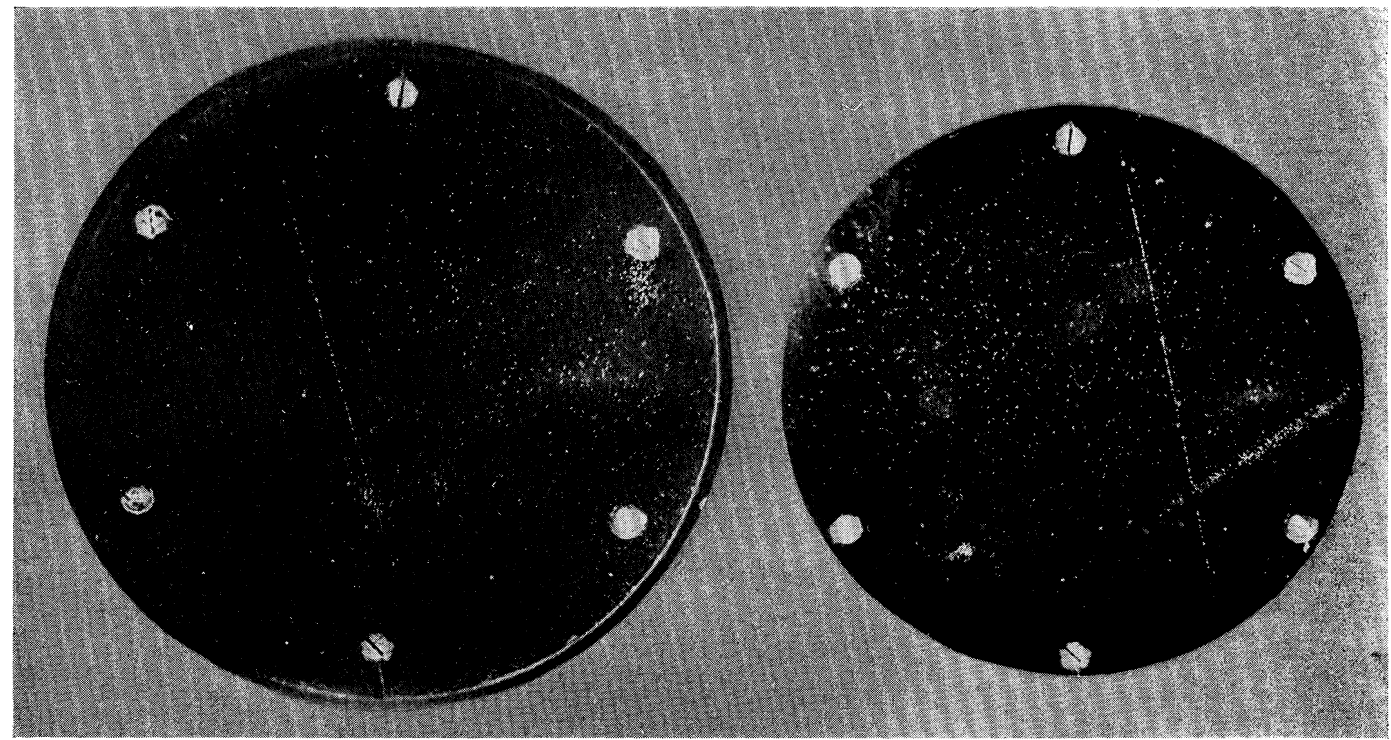

FIG. 14. A positron of 900 million volts energy.

FIG. 15. An electron of 1300 million volts energy.

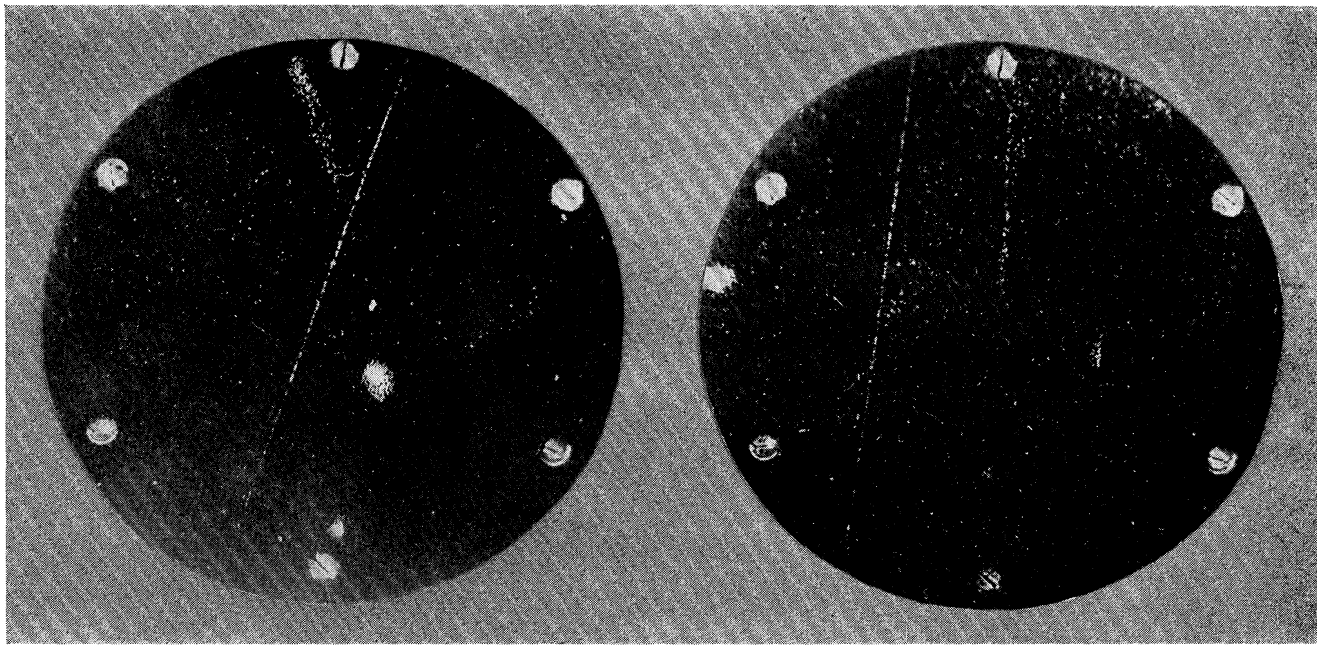

FIG. 16. A positron of 2700 million volts energy.

FIG. 17. An electron of 5000-7000 million volts energy. 


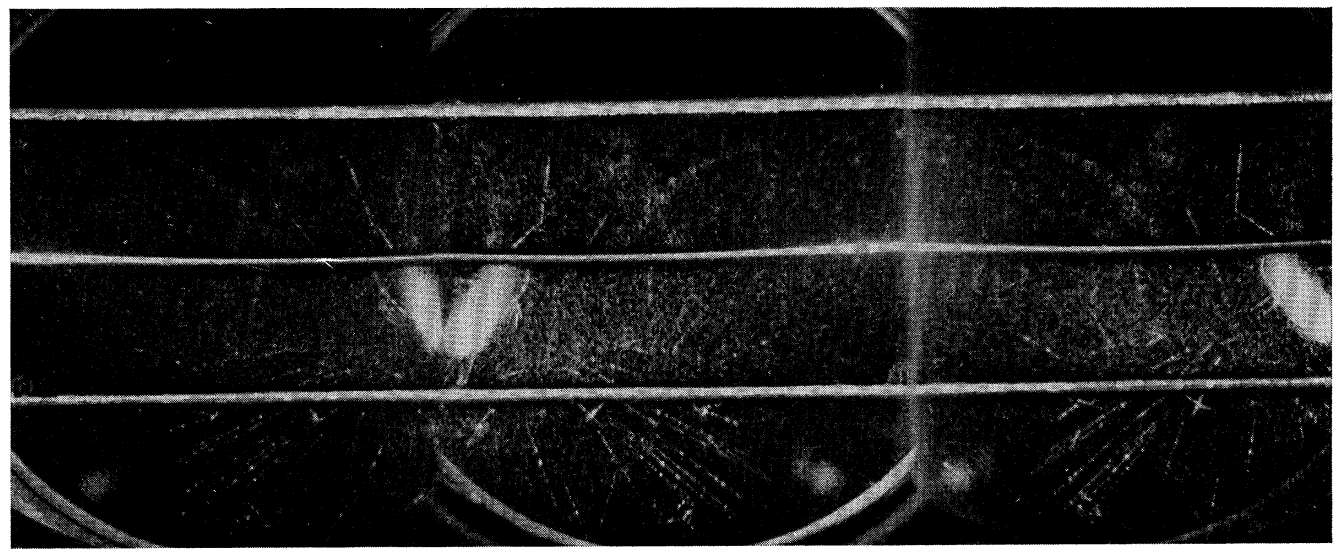

FIG. 18. A shower of more than 20 cosmic-ray particles originating in the iron of the pole piece. Three views are shown, the outer two views are mirror images of the direct central view. The outer views are equivalent to photographs taken by cameras placed on either side of the chamber with their axes inclined at 12.5 degrees to the axis of the chamber. This shower occurred during a study of the Th $C^{\prime \prime}$ gamma-ray effects and the magnetic field was adjusted to the low value of 650 gauss. Although most of the tracks are essentially straight both positives and negatives occur, and their energies are all above those to be attributed to effects of the gamma-rays present. The short, thick track is very probably an alpha-particle arising from stray radioactive material in the chamber. Its high degree of diffuseness indicates that it passed through the chamber before the shower of cosmic-ray particles occurred.

our data are based on too few tracks to detect a small difference in number between positives and negatives, the excess of high energy positives over negatives agrees in a general way with the tube-counter experiments of Johnson ${ }^{14}$ and Alvarez and Compton, ${ }^{15}$ dealing with large numbers of particles, which show an east-west asymmetry that indicates an excess of positives over negatives.

A certain group of 815 tracks most of which were not capable of accurate measurement for energy were classified as to multiplicity of tracks with the following results: there occurred 708 singles, 82 doubles, 7 triples, 7 quartets, 3 quintets, 7 having between 6 and 10 tracks, and one with more than 10 . Photographs showing more than one track were not counted as examples of multiple tracks unless the tracks were clearly associated in time as shown by an equality in diffusion of the ions on all the tracks. In most cases the multiple tracks appear to originate at a common center, but in some cases there also occur tracks definitely associated in time but apparently not coming from the same source. As a general rule in the photographs

${ }^{14}$ Johnson, Phys. Rev. 43, 834 (1933).

15 Alvarez and Compton, Phys. Rev. 43, 835 (1933). showing several tracks both positives and negatives occur.

\section{ConCluding Discussion}

To obtain a complete description of the primary cosmic-ray beam it will of course be necessary to understand in detail the absorption processes involved. Experiments have shown that these processes are of considerable complexity and further experimental data are needed. Certain general conclusions, however, can perhaps safely be drawn from the experimental data at hand. We shall consider the primary cosmicray beam at sea level to consist in greater part of photons, a point of view held by Professor Millikan for several years, and now given additional support by the fact that hard gamma-rays of Th $C^{\prime \prime}$ have been found to produce positrons as do the cosmic rays. ${ }^{16},{ }^{17}$ It is apparent at once as we have already pointed out that the KleinNishina formula which deals only with absorption by free electrons cannot be valid for radiation as energetic as the cosmic rays. ${ }^{2}$ To explain the presence of the large number of positrons

\footnotetext{
${ }^{16}$ Anderson, Science 77, 432 (1933).

${ }^{17}$ Anderson and Neddermeyer, Phys. Rev. 43, 1034 (1933).
} 
observed, and their frequent occurrence in groups associated with negative electrons, it is necessary to call upon a new type of absorption which must be due to nuclear effects.

The excess absorption ${ }^{18}$ and anomalous scattering ${ }^{19}$ effects of Th $C^{\prime \prime}$ gamma-rays in the heavy elements is undoubtedly due at least in part to the production and perhaps the annihilation of positrons and amounts to approximately 20 percent of the total absorption for the hard component $\left(2.6 \times 10^{6}\right.$ volts $)$ of Th $C^{\prime \prime}$ gamma-rays in lead. In contrast to the approximately equal numbers of positives and negatives for the cosmic-ray particles we have observed a considerably greater number of negatives than positives ejected from lead by the Th $C^{\prime \prime}$ rays. This ac-

${ }^{18}$ Chao, Proc. Nat. Acad. Sci. 16, 431 (1930); Phys. Rev. 36, 1519 (1930); and Meitner and Hupfield, Naturwiss. 19, 775 (1931).

${ }^{19}$ Gray and Tarrant, Proc. Roy. Soc. A136, 662 (1932). cords with the view that absorption by the free negative electrons represents the more important part of the absorption for gamma-rays of this energy. A statistical study of the positives and negatives produced by $T h C^{\prime \prime}$ gamma-rays is in progress.

The striking fact that in the cosmic rays positives and negatives occur in practically equal numbers and have apparently a similar energy distribution is in accord with the assumption that the nuclear effects involved give rise to the positives and negatives in pairs (in some cases several pairs as evidenced by the showers), and that this type of absorption represents nearly the whole absorption for rays in the energy range of hundreds of millions of volts, the absorption by free electrons being of relatively minor importance.

I wish to make grateful acknowledgment to the Carnegie Corporation for a grant which made this work possible and to Mr. Seth H. Neddermyer for his assistance. 


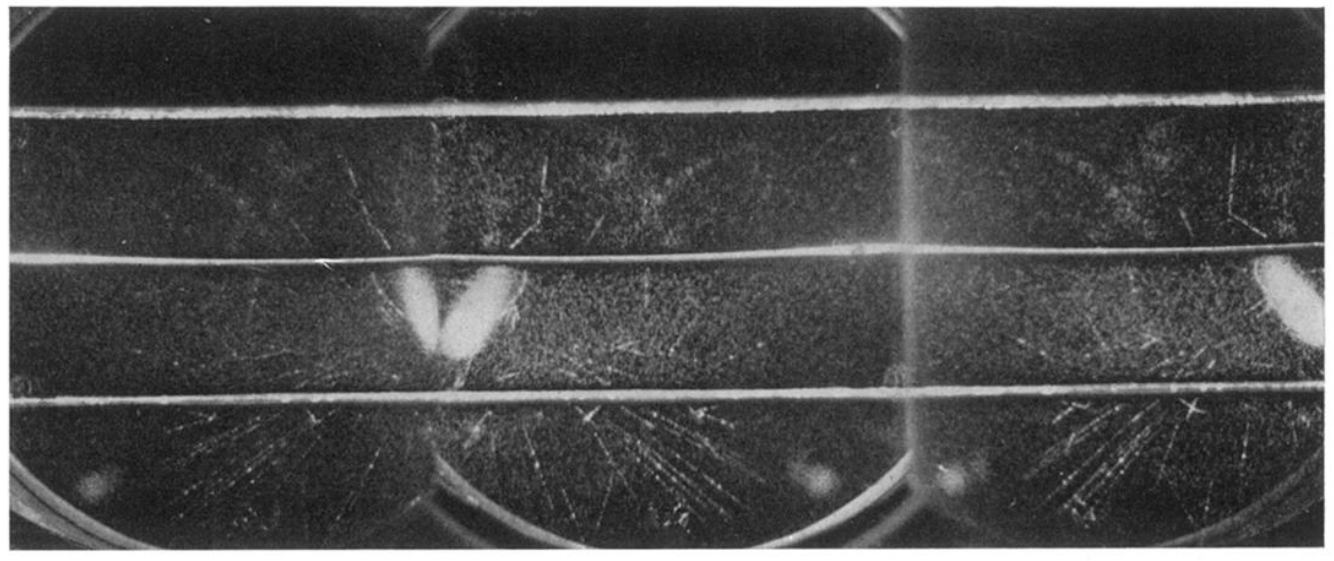

FIG. 18. A shower of more than 20 cosmic-ray particles originating in the iron of the pole piece. Three views are shown, the outer two views are mirror images of the direct central view. The outer views are equivalent to photographs taken by cameras placed on either side of the chamber with their axes inclined at 12.5 degrees to the axis of the chamber. This shower occurred during a study of the Th $C^{\prime \prime}$ gamma-ray effects and the magnetic field was adjusted to the low value of 650 gauss. Although most of the tracks are essentially straight both positives and negatives occur, and their energies are all above those to be attributed to effects of the gamma-rays present. The short, thick track is very probably an alpha-particle arising from stray radioactive material in the chamber. Its high degree of diffuseness indicates that it passed through the chamber before the shower of cosmic-ray particles occurred. 


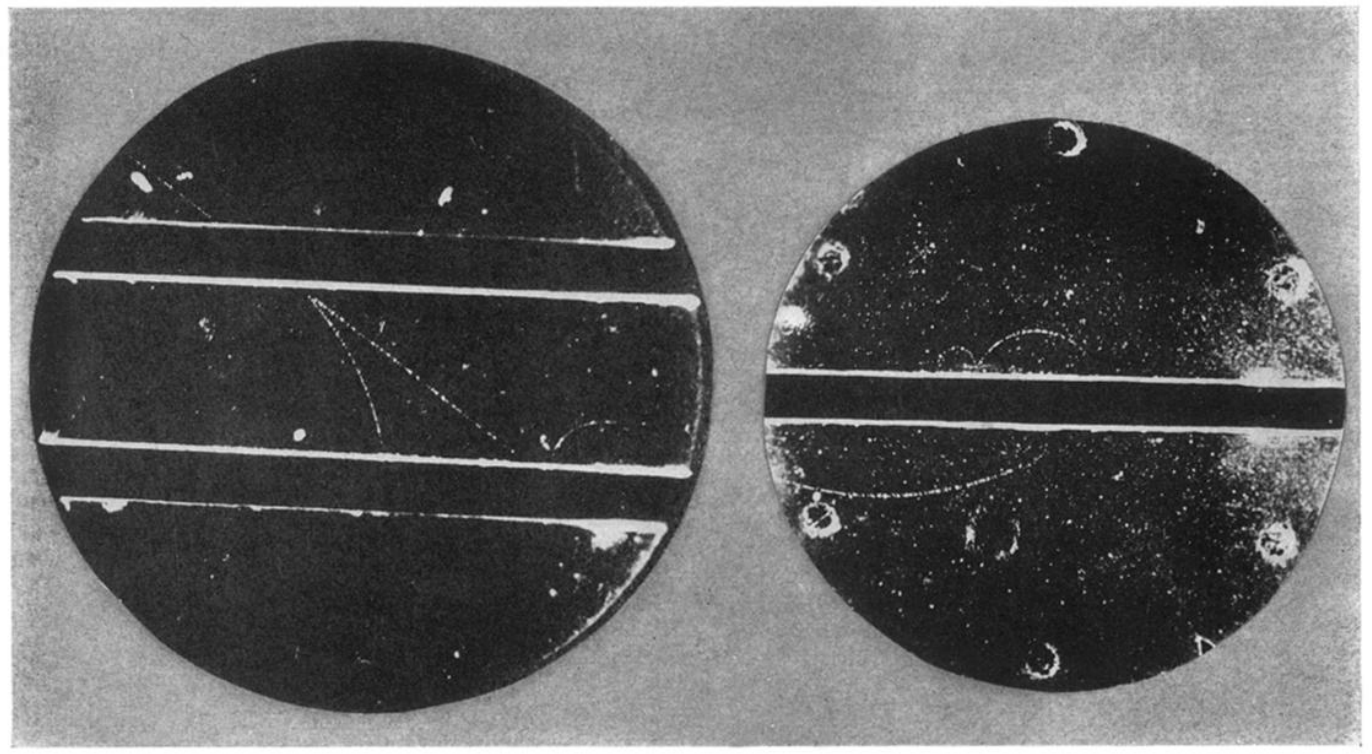

10

11

FIG. 10. A secondary electron of 32 million volts energy was ejected by a fast electron which passed through the lead plate. The angle, $\theta$, between the directions of the primary and secondary electrons satisfies within experimental uncertainty the relation $\tan \theta=\left(2 m c^{2} / E\right)^{\frac{1}{2}}$, where $E$ is the energy of the secondary electron. The energy of the primary electron measured between the plates is about 800 million volts. An additional secondary electron of about 6 million volts energy was produced in the lower plate and appears just above the lower plate at the right. The frequency of occurrence of secondary electrons produced by close encounters is in close agreement with that to be expected from theoretical considerations.

FIG. 11. There are two interpretations of this photograph. The first is that a positron of 38 million volts energy penetrated the lead plate and emerged above with 6 million volts energy. It is seen to suffer a sharp deflection upon striking the upper surface of the lead plate. The second interpretation is that an electron of 38 million volts energy was ejected downward from the plate, and at the same time a positron of 6 million volts energy ejected upward and deflected by the magnetic field until it struck the plate and rebounded. Either of these two views requires the positron interpretation. 


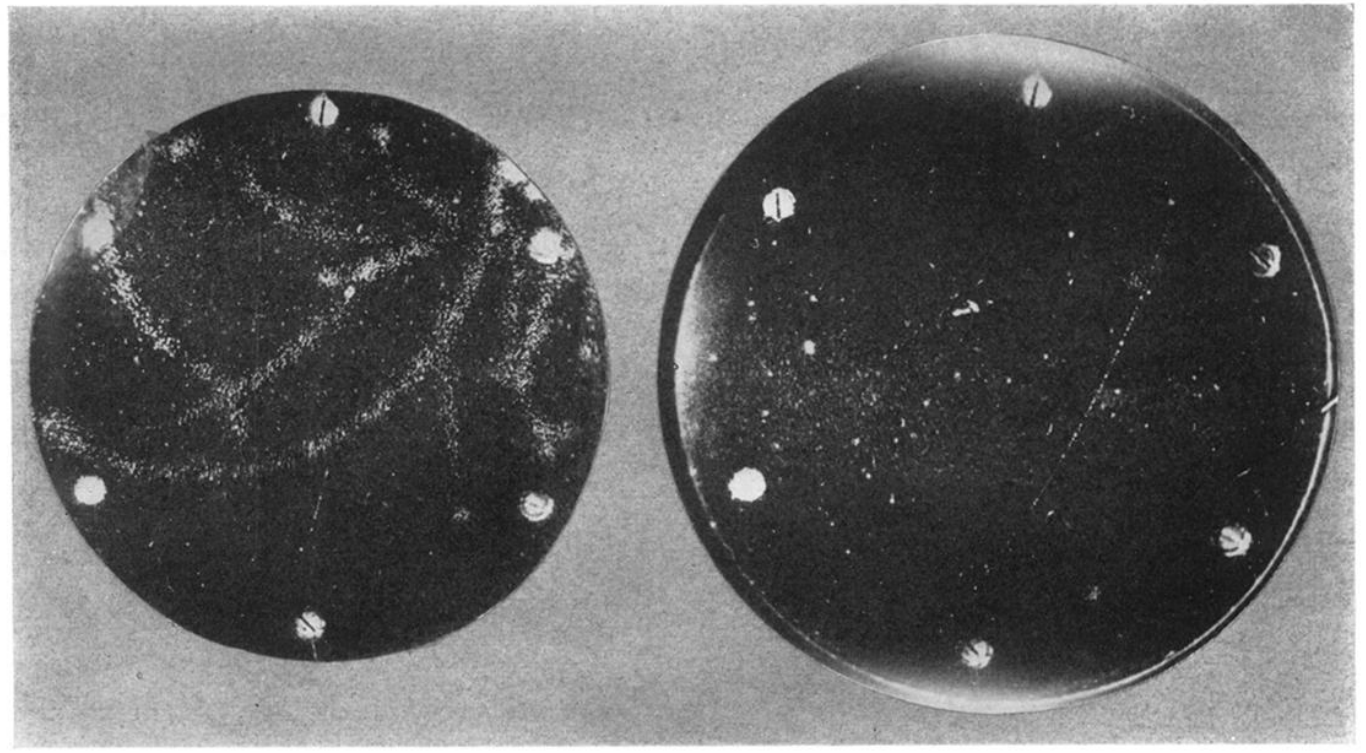

12

FIG. 12. A shower represented by a group of simultaneous early tracks. From the specific ionization and curvature in the magnetic field it is necessary to ascribe most of these tracks to particles of small mass, i.e., positrons or electrons.

FIG. 13. An electron of 120 million volts energy. 


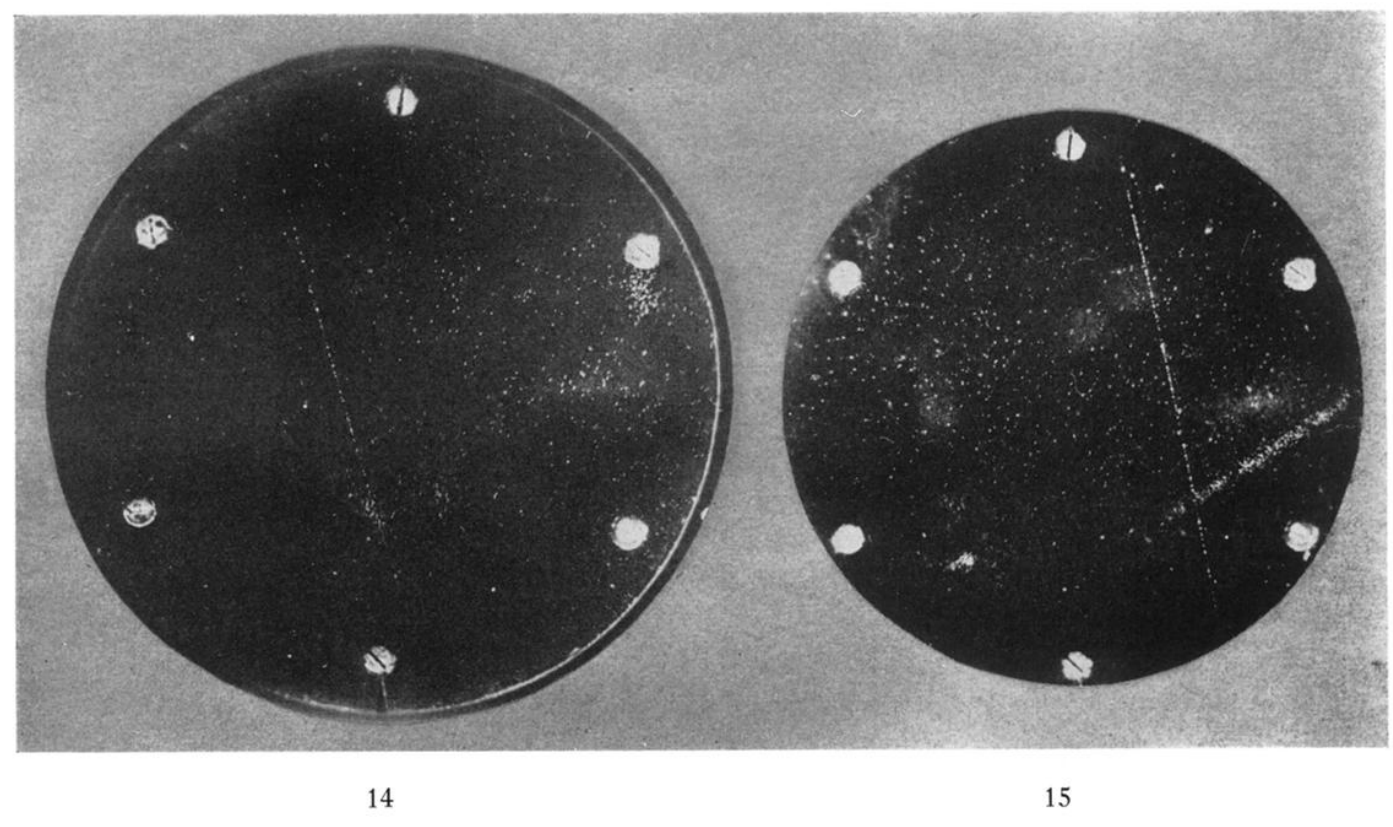

FIG. 14. A positron of 900 million volts energy.

FIG. 15. An electron of 1300 million volts energy. 


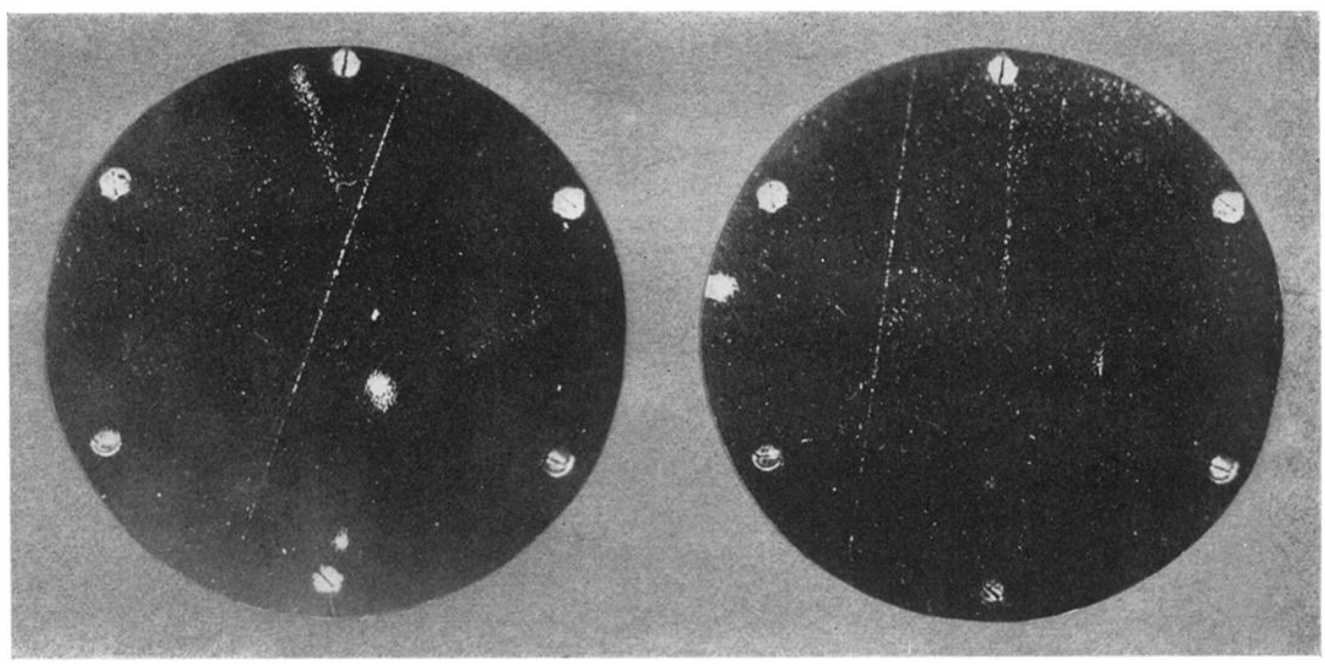

16

17

FIG. 16. A positron of 2700 million volts energy.

FIG. 17. An electron of 5000-7000 million volts energy. 


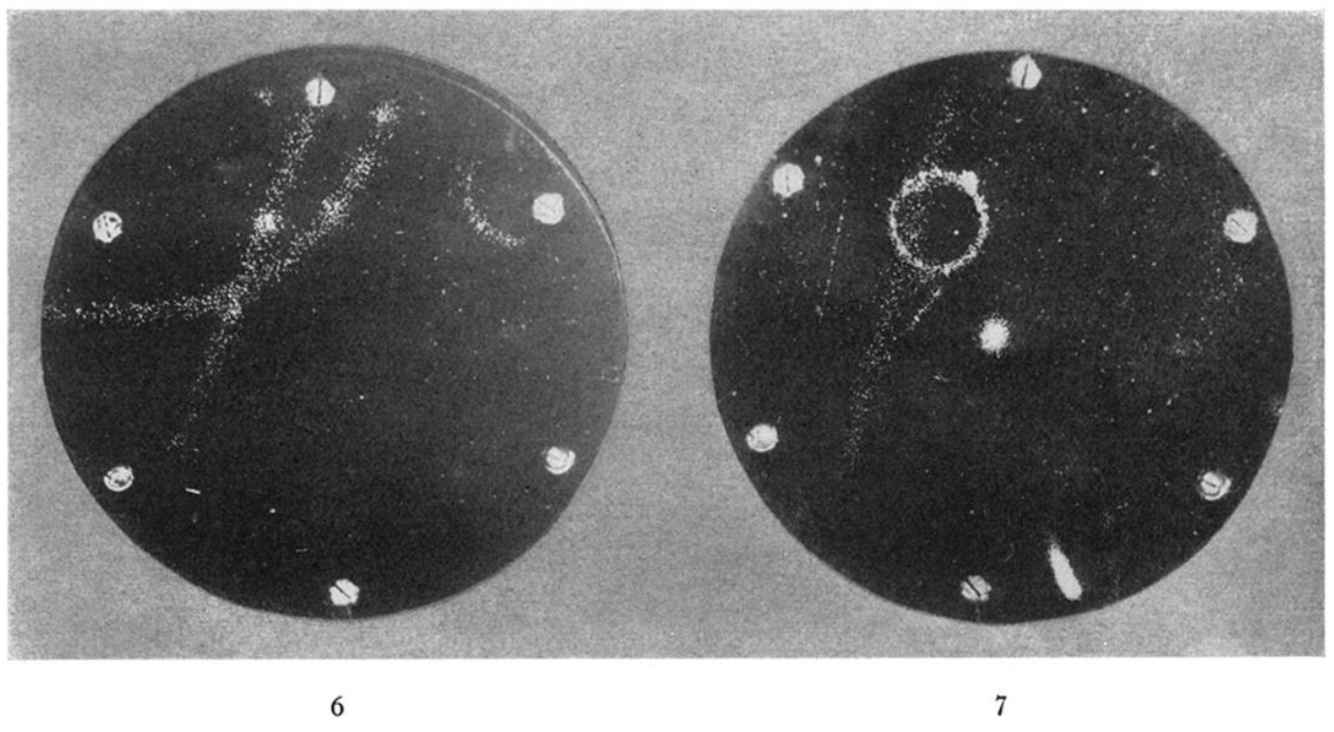

FIG. 6. A representative group of three "early" tracks suitable for an ion-count, showing drops resolved as a result of the diffusion of the ions. On the upper portion of the high energy track is a small cluster of ions.

FIG. 7. Three ages of tracks are represented: (1) a high energy very diffuse early track, (2) a later pair of tracks showing less diffusion of ions; the circular track is an electron of 4.8 million volts energy, the other one a higher energy track of uncertain sign of charge, and (3) at the left a short, sharp track produced by a particle which entered the chamber coincident with or immediately following the expansion. 


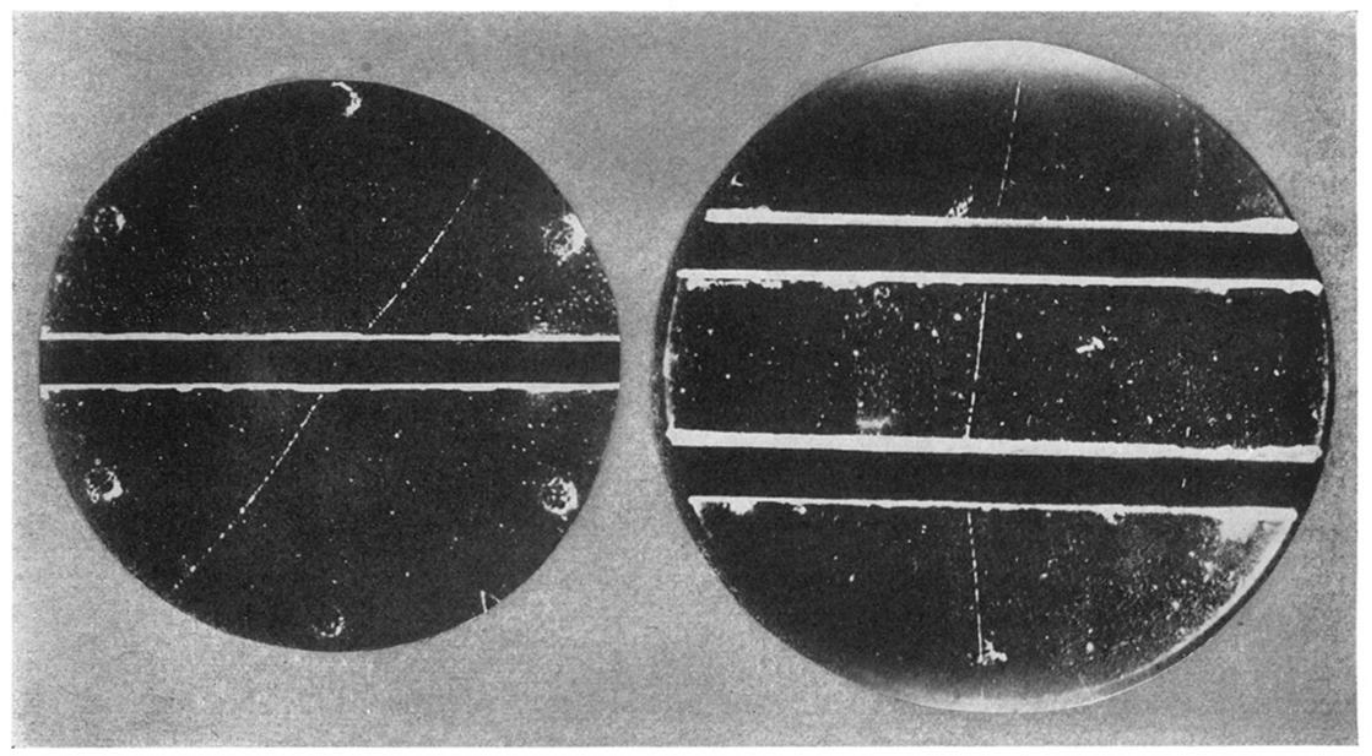

8

9

FIG. 8. A 113 million volt-electron traversed $13.4 \mathrm{~mm}$ of lead and emerged with an energy of 86 million volts, showing an energy loss of 20 million volts per $\mathrm{cm}$ of lead. Thickness of lead plate is $11 \mathrm{~mm}$.

FIG. 9. An electron of 240 million volts energy lost about 20 million volts in first passage through the $11 \mathrm{~mm}$ lead plate and an additional 60 million volts in the lower plate. 